\title{
Displaying All Bangla Compound Letters \& Alphabets By 32- Segment
}

\author{
Shamsun Nahar ${ }^{1}$, Fariha Jabin ${ }^{2}$ \\ ${ }^{1}$ International Islamic University Chittagong, Bangladesh \\ ${ }^{2}$ Jahangirnagar University, Bangladesh
}

\begin{abstract}
Different approaches have been proposed for representing Bangla and English alphabets and numerals by segment display. But there is no complete and accurate scheme has been done yet for Bangla compound letters. The focus of our paper is on all compound letters to represent by 32 segments. By these 32 segments, we also represent all Bengali and English numerals and alphabets. Accurate recognition of compound characters is difficult due to their complex shapes. In this paper we try to present total 97 compound letters in simpler look. This proposed design performs well for complex-shaped compound characters, which were confusing to the existing methods.
\end{abstract}

Keywords: 32-segment display, alphabets, compound letters and numerals.

\section{INTRODUCTION}

The representation of Bengali compound letters is an interesting task on which several researchers have been done for recognizing Bengali simple characters. But, some of them suffer from poor display of the shapes of the characters and some designs are difficult to implement. There are 11 vowels and 29 consonants in Bangla.

For display Bangla numerals several works as like 9 segment, 10 segment,11 segment,12 segmented display [1],[2],[3],[4],[5],[6] are already proposed. Few researchers' already worked on Bangla vowels by using 14 and 17 segments [7], [8]. We found 44 segments just represented all Bangla consonant [9]. Another paper of 24 segments represented numeral, vowel and some consonant [10]. Some researchers designed 31 segments for few consonant and numeral [11]. One more paper shows 32 segments for all Bangla alphabets and numeral [12]. Another paper works on 26 segments for all Bangla alphabets [13]. We also found 34 segments for all Bangla and English alphabets and numeral [14]. Only one paper stands for some Bangla compound letters (but no vowel \& consonant) by using 36 segments [15]. There is also a paper of $n$-segment [16] display for Bangla alphabets.

\section{Method}

In this paper we try to draw a segmented display for Bangla alphabets, compound letters, numerals and also English alphabets and numerals, that's why firstly we have listed all compound letters [17] and found 97 available useable compound letters for Bangla. Our 32 segmented displays can show all of them. Here we show অ but do not show the $2^{\text {nd }}$ alphabet আ because when we use the vowel sign (স্বরচিহ্) ) with our proposed design it can be easily displayed. Here the figure 1 shows 32 -segmented display.

Figure 1: proposed 32 segmented display

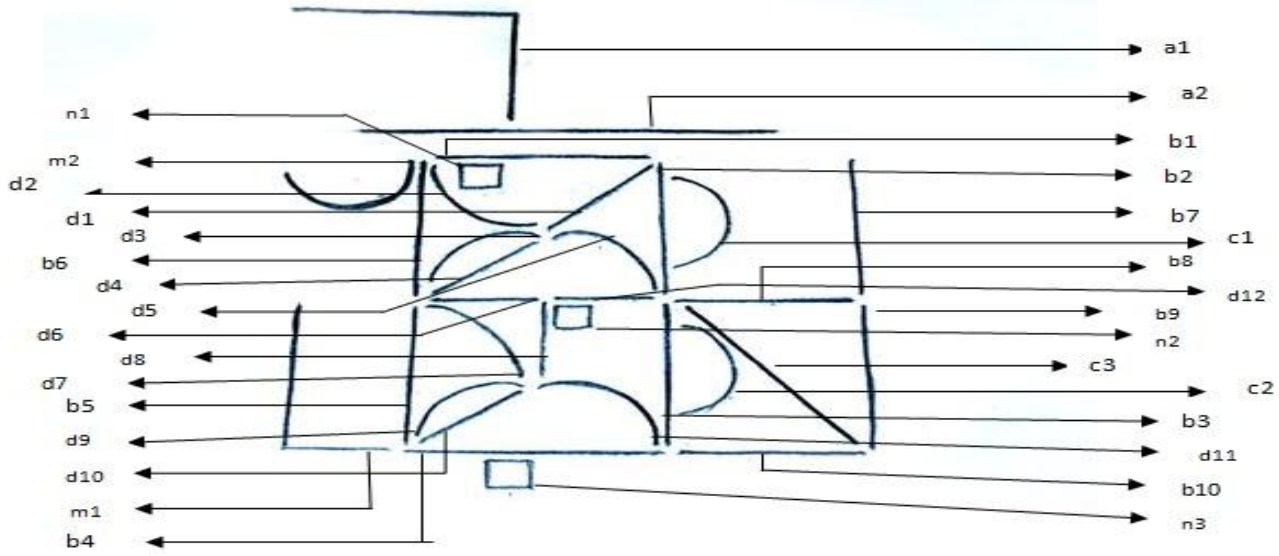

In fig. 2 we show Bangla vowel and consonant by using this 32 segmented display. 


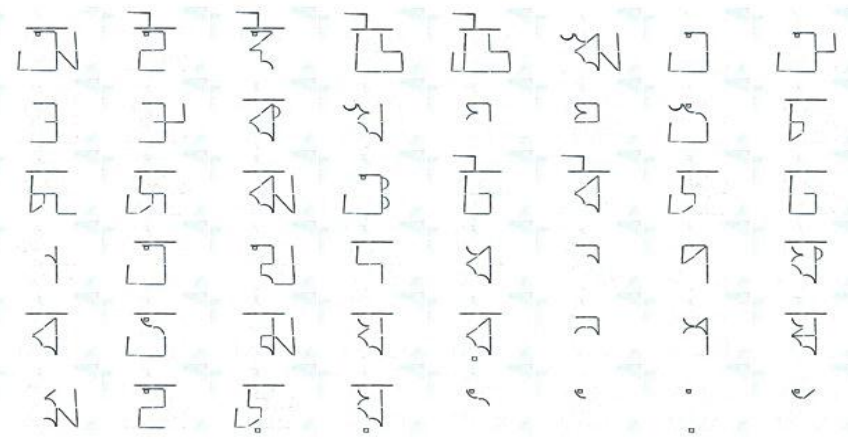

Figure 2: Bangla vowel and consonant by using 32 segment displays

We found 97 compound letters and designed them by 32 segment display. Mainly we collect compound letter list from Bangla Academy Dictionary, there are some compound letters which have no use now a days, that's why by using Bangla font software (Avro, Bijoy) we cannot write them combined but we also design segmented display for them as like ডগ, নঢ. The list of all compound letters shown on table 1.

Table 1: 97 Compound Letters And Their Segment List

\begin{tabular}{|c|c|c|c|c|c|}
\hline no & $\begin{array}{l}\text { compo } \\
\text { und } \\
\text { letters }\end{array}$ & segment numbers & no & $\begin{array}{l}\text { compo } \\
\text { und } \\
\text { letters }\end{array}$ & segment numbers \\
\hline 1. & 雨 & $\mathrm{d} 1, \mathrm{~d} 5, \mathrm{~b} 2, \mathrm{c} 1, \mathrm{~d} 12, \mathrm{~d} 8, \mathrm{~d} 11, \mathrm{c} 2, \mathrm{~b} 3, \mathrm{a} 2$ & 25. & प্ড & $\mathrm{a} 2, \mathrm{~d} 1, \mathrm{~d} 5, \mathrm{~d} 12, \mathrm{~d} 6, \mathrm{~b} 6, \mathrm{~d} 8, \mathrm{~d} 11, \mathrm{~b} 4, \mathrm{~b} 5$ \\
\hline 2. & ক্ট & b1,d1,d5,b2,c1,b3,b10,b9,a1 & 26. & ডগ & $\mathrm{a} 2, \mathrm{~d} 1, \mathrm{~d} 5, \mathrm{~d} 12, \mathrm{~d} 6, \mathrm{~b} 6, \mathrm{~d} 7, \mathrm{~d} 10, \mathrm{~b} 3$ \\
\hline 3. & $\overline{3}$ & a2,b1,b2,d12,b3,b4,b8,b9 & 27. & न्ট & $\mathrm{a} 2, \mathrm{~d} 5, \mathrm{~b} 2, \mathrm{a} 1, \mathrm{~b} 3, \mathrm{~b} 10, \mathrm{~b} 9$ \\
\hline 4. & ক্ল & $\mathrm{b} 1, \mathrm{~b} 2, \mathrm{~d} 1, \mathrm{~d} 5, \mathrm{c} 1, \mathrm{~d} 9, \mathrm{~d} 11, \mathrm{~b} 3$ & 28. & न्ड & a2,d5,b2,d8,d11,b4,b5 \\
\hline 5. & ফ & $\begin{array}{l}\text { b1,d2,d4,d3,d7,d10,d9,d11,d3,d2, } \\
\text { c1 }\end{array}$ & 29. & নঢ & a2,d5,b2,b3,b10,b9 \\
\hline 6. & क्म & a2,b2,d1,d5,c1,b7,b9,b10,d11 & 30. & ন্ত & $\mathrm{a} 2, \mathrm{~d} 5, \mathrm{~b} 2, \mathrm{n} 2, \mathrm{~d} 12, \mathrm{~b} 3, \mathrm{~b} 4, \mathrm{~b} 5$ \\
\hline 7. & গ્ন & $\mathrm{d} 2, \mathrm{~d} 4, \mathrm{~b} 1, \mathrm{~b} 2, \mathrm{~b} 3, \mathrm{~d} 11$ & 31. & न्ब्र & $\mathrm{a} 2, \mathrm{~d} 5, \mathrm{~b} 2, \mathrm{n} 2, \mathrm{~d} 12, \mathrm{~b} 3, \mathrm{~b} 4, \mathrm{~m} 1$ \\
\hline 8. & গ্ধ & $\mathrm{d} 2, \mathrm{~d} 4, \mathrm{~b} 1, \mathrm{~b} 2, \mathrm{~d} 12, \mathrm{~d} 8, \mathrm{~d} 11, \mathrm{~b} 3, \mathrm{~b} 8, \mathrm{~b} 9$ & 32. & ন্থ & $\mathrm{a} 2, \mathrm{~d} 5, \mathrm{~b} 2, \mathrm{n} 2, \mathrm{~b} 3, \mathrm{~b} 10, \mathrm{~b} 9, \mathrm{~b} 7$ \\
\hline 9. & গ & $\mathrm{b} 1, \mathrm{~d} 2, \mathrm{~d} 4, \mathrm{~d} 12, \mathrm{~d} 8, \mathrm{~d} 11, \mathrm{~b} 3, \mathrm{c} 3, \mathrm{~b} 7$ & 33. & न्म & $\mathrm{a} 2, \mathrm{~d} 5, \mathrm{~b} 2, \mathrm{~b} 8, \mathrm{~b} 9$ \\
\hline 10. & গ્ল & $\mathrm{b} 1, \mathrm{~d} 2, \mathrm{~d} 4, \mathrm{~d} 9, \mathrm{~d} 11, \mathrm{~b} 3$ & 34. & ন্ধ & $\mathrm{a} 2, \mathrm{~d} 5, \mathrm{~b} 2, \mathrm{~d} 12, \mathrm{~d} 8, \mathrm{~d} 11, \mathrm{~b} 3, \mathrm{~b} 8, \mathrm{~b} 9$ \\
\hline 11. & ঙ্ক & $\begin{array}{l}\mathrm{m} 2, \mathrm{~b} 6, \mathrm{~d} 6, \mathrm{~d} 12, \mathrm{~d} 5, \mathrm{~d} 2, \mathrm{n} 1, \mathrm{~d} 8, \mathrm{~d} 11, \mathrm{~b} 3, \\
\mathrm{c} 2\end{array}$ & 35. & ন্ন & $\mathrm{a} 2, \mathrm{~d} 5, \mathrm{~b} 2, \mathrm{~d} 11, \mathrm{~b} 3$ \\
\hline 12. & झ्ञ & $\begin{array}{l}\text { m2,b6,d6,d12,d5,d2,n1,d7,d8,d11, } \\
\text { b3 }\end{array}$ & 36. & ন্ম & $\mathrm{a} 2, \mathrm{~d} 5, \mathrm{~b} 2, \mathrm{~d} 12, \mathrm{~d} 8, \mathrm{~d} 11, \mathrm{~b} 3, \mathrm{c} 3, \mathrm{~b} 9, \mathrm{~b} 7$ \\
\hline 13. & $\check{\Upsilon}$ & $\mathrm{m} 2, \mathrm{~b} 6, \mathrm{~d} 6, \mathrm{~d} 12, \mathrm{~d} 5, \mathrm{~d} 2, \mathrm{n} 1, \mathrm{~d} 7, \mathrm{~d} 10, \mathrm{~b} 3$ & 37. & $\overline{3}$ & a2,b1,b2,d12,b3,b4 \\
\hline 14. & ষ্ম & $\begin{array}{l}\mathrm{m} 2, \mathrm{~b} 6, \mathrm{~d} 6, \mathrm{~d} 12, \mathrm{~d} 5, \mathrm{~d} 2, \mathrm{n} 1, \mathrm{~d} 7, \mathrm{~d} 10, \mathrm{~d} 9, \\
\mathrm{~d} 11, \mathrm{~b} 3\end{array}$ & 38. & $\bar{ন}$ & a2,n1,b1,b2,d12,d6,b6,d11,b3 \\
\hline 15. & ष & $\mathrm{a} 2, \mathrm{~b} 6, \mathrm{~d} 6, \mathrm{~d} 12, \mathrm{~b} 2, \mathrm{c} 1, \mathrm{~b} 5, \mathrm{~b} 4, \mathrm{~b} 3$ & 39. & $\bar{্}$ & a2,n1,b1,b2,d12,d6,b6,d8,d11,b3 \\
\hline 16. & ष्क & $\mathrm{a} 2, \mathrm{~b} 6, \mathrm{~d} 6, \mathrm{~d} 12, \mathrm{~b} 2, \mathrm{c} 1, \mathrm{~b} 5, \mathrm{~b} 4, \mathrm{~b} 3, \mathrm{c} 3$ & 40. & অ & $\begin{array}{l}\text { a2,n1,b1,b2,d12,d6,b6,d8,d11,b3,c } \\
3, \mathrm{~b} 9, \mathrm{~b} 7\end{array}$ \\
\hline 17. & জ্জ & $\begin{array}{l}\mathrm{d} 1, \mathrm{~d} 5, \mathrm{~d} 6, \mathrm{~d} 12, \mathrm{c} 1, \mathrm{~b} 6, \mathrm{~b} 5, \mathrm{~b} 4, \mathrm{~d} 11, \mathrm{~d} 8, \\
\mathrm{c} 2\end{array}$ & 41. & থ & $\mathrm{d} 2, \mathrm{~d} 4, \mathrm{~d} 6, \mathrm{~d} 12, \mathrm{~d} 7, \mathrm{~d} 10, \mathrm{~b} 4, \mathrm{~b} 3$ \\
\hline 18. & ङ্ঝ & $\begin{array}{l}\text { d1,d5,d6,d12,c1,b6,d8,d11,b3,c3, } \\
\text { b9 }\end{array}$ & 42. & দ⿹ & $\mathrm{b} 6, \mathrm{~d} 4, \mathrm{~d} 5, \mathrm{~d} 6, \mathrm{~d} 12, \mathrm{~d} 7, \mathrm{~d} 9, \mathrm{~b} 3$ \\
\hline 19. & छ্ব & $\mathrm{d} 1, \mathrm{~d} 5, \mathrm{~d} 6, \mathrm{~d} 12, \mathrm{c} 1, \mathrm{~b} 6, \mathrm{~d} 9, \mathrm{~d} 11, \mathrm{b3}$ & 43. & $\bar{\eta}$ & $\mathrm{a} 2, \mathrm{~b} 6, \mathrm{~d} 6, \mathrm{~d} 8, \mathrm{~d} 11$ \\
\hline 20. & $\bar{\Xi}$ & $\mathrm{d} 1, \mathrm{~d} 5, \mathrm{~b} 3, \mathrm{~b} 4, \mathrm{c} 1, \mathrm{c} 2, \mathrm{~m} 1$ & 44. & দ্ধ & a2,b6,d6,d12,d8,d11,b3,b8,b9 \\
\hline 21. & 官 & $\mathrm{a} 2, \mathrm{~d} 5, \mathrm{~b} 2, \mathrm{~d} 8, \mathrm{~d} 11, \mathrm{~b} 3, \mathrm{c} 1, \mathrm{c} 2$ & 45. & দ & $\mathrm{a} 2, \mathrm{~b} 6, \mathrm{~d} 6, \mathrm{~d} 12, \mathrm{~d} 8, \mathrm{~d} 11, \mathrm{~b} 3$ \\
\hline 22. & 3 & $\mathrm{a} 2, \mathrm{~d} 5, \mathrm{~b} 2, \mathrm{~d} 8, \mathrm{~d} 11, \mathrm{~b} 4, \mathrm{~b} 5, \mathrm{c} 1, \mathrm{c} 2, \mathrm{c} 3$ & 46. & দ⿹ & a2,b6,d6,d8,n2,d11,b4,b5 \\
\hline 23. & $\dddot{q}$ & $\begin{array}{l}\text { a2,d5,b2,d12,d8,d11,c1,c2,c3,b9,b } \\
7\end{array}$ & 47. & দ্ & a2,b6,d6,d8,d11,b3,d12,c3,b9,b7 \\
\hline 24. & ট & $\mathrm{a} 1, \mathrm{a} 2, \mathrm{~b} 4, \mathrm{~b} 5, \mathrm{~b} 6, \mathrm{~d} 8, \mathrm{~d} 10, \mathrm{~d} 11$ & 48. & ধ্ব & $\mathrm{d} 1, \mathrm{~d} 2, \mathrm{~d} 5, \mathrm{~b} 2, \mathrm{~d} 12, \mathrm{~d} 8, \mathrm{~d} 11, \mathrm{~b} 3$ \\
\hline no & compo & segment numbers & no & compo & segment numbers \\
\hline
\end{tabular}




\begin{tabular}{|c|c|c|c|c|c|}
\hline & $\begin{array}{c}\text { und } \\
\text { letters }\end{array}$ & & & $\begin{array}{c}\text { und } \\
\text { letters }\end{array}$ & \\
\hline 49. & ম্ট & b1,b6,d4,d1,b2,b3,b10,b9 & 74. & ल्ल & $\mathrm{d} 3, \mathrm{~d} 5, \mathrm{~b} 2, \mathrm{a} 2, \mathrm{~d} 9, \mathrm{~d} 11, \mathrm{~b} 3$ \\
\hline 50. & প্তু & b1,b6,d4,d1,b2,n2,d12,b3,b4,b5 & 75. & 2 & $\mathrm{d} 2, \mathrm{~d} 3, \mathrm{~d} 5, \mathrm{~d} 1, \mathrm{~b} 2, \mathrm{~b} 3, \mathrm{c} 2$ \\
\hline 51. & প্প & b1,b6,d4,d1,b2,d6,d12,d7,d8,b3, & 76. & ख्र & $\mathrm{d} 2, \mathrm{~d} 3, \mathrm{~d} 5, \mathrm{~d} 1, \mathrm{~b} 2, \mathrm{~b} 3, \mathrm{c} 2, \mathrm{~b} 10$ \\
\hline 52. & প্ল & b1,b6,d4,d1,b2,d9,d11,b3 & 77. & শ્ન & $\mathrm{d} 2, \mathrm{~d} 3, \mathrm{~d} 5, \mathrm{~d} 1, \mathrm{~b} 2, \mathrm{~d} 11, \mathrm{~b} 3$ \\
\hline 53. & म & b1,b6,d4,d1,b2,d7,d10,d11,b3 & 78. & শ্ব & $\mathrm{d} 2, \mathrm{~d} 3, \mathrm{~d} 5, \mathrm{~d} 1, \mathrm{~b} 2, \mathrm{~d} 12, \mathrm{~d} 8, \mathrm{~d} 11, \mathrm{~b} 3$ \\
\hline 54. & ব্জ & b1,d1,d5,b2,d12,d8,d11,b4,b5,b3 & 79. & শ্ম & $\begin{array}{l}\text { d2,d3,d5,d1,b2,d12,d8,d11,b3,c3,b } \\
9, \mathrm{~b} 7\end{array}$ \\
\hline 55. & ব্দ & b1,d1,d5,b2,b8,b9 & 80 . & ক্ক & $\mathrm{d} 2, \mathrm{~d} 3, \mathrm{~d} 5, \mathrm{~d} 6, \mathrm{~d} 12, \mathrm{~b} 2, \mathrm{~d} 8, \mathrm{~d} 11, \mathrm{~b} 3, \mathrm{c} 2$ \\
\hline 56. & ব্ধ & b1,d1,d5,b2,d12,d8,d11,b3,b8,b9 & 81. & ষ্ট & d2,d3,d5,d6,d12,b2,b3,b10,b9,a1 \\
\hline 57. & ব্ল & $\mathrm{b} 1, \mathrm{~d} 1, \mathrm{~d} 5, \mathrm{~b} 2, \mathrm{~d} 9, \mathrm{~d} 11, \mathrm{~b} 3$ & 82. & ষ্ণ & $\mathrm{d} 2, \mathrm{~d} 3, \mathrm{~d} 6, \mathrm{~d} 12, \mathrm{~b} 2, \mathrm{~d} 7, \mathrm{~d} 11, \mathrm{~b} 3, \mathrm{c} 1, \mathrm{c} 2$ \\
\hline 58. & ন্ব & $\mathrm{b} 1, \mathrm{~d} 1, \mathrm{~d} 5, \mathrm{~b} 2, \mathrm{~d} 12, \mathrm{~d} 8, \mathrm{~d} 11, \mathrm{~b} 3$ & 83. & क्भ & $\mathrm{d} 2, \mathrm{~d} 3, \mathrm{~d} 5, \mathrm{~d} 6, \mathrm{~d} 12, \mathrm{~b} 2, \mathrm{~d} 7, \mathrm{~d} 3, \mathrm{~b} 3$ \\
\hline 59. & বভ & b1,d1,d5,b2,n2,d8,d11,b4,b5 & 84. & क्य & $\mathrm{d} 2, \mathrm{~d} 3, \mathrm{~d} 5, \mathrm{~d} 6, \mathrm{~d} 12, \mathrm{~b} 2, \mathrm{~d} 11, \mathrm{~b} 3, \mathrm{c} 1, \mathrm{~d} 7$ \\
\hline 60. & म্न & $\mathrm{d} 2, \mathrm{~d} 3, \mathrm{~d} 4, \mathrm{~d} 5, \mathrm{~b} 2, \mathrm{~d} 11, \mathrm{~b} 3$ & 85. & ब্ম & $\begin{array}{l}\text { d2,d3,d5,d6,d12,b2,d8,d11,b3,c3,b9,b } \\
7\end{array}$ \\
\hline 61. & मभ & $\begin{array}{l}\text { d2,d3,d4,d5,b2,d12,d8,d11,b3,b8, } \\
\text { b9 }\end{array}$ & 86. & স্ক & $\mathrm{d} 2, \mathrm{~d} 4, \mathrm{~d} 5, \mathrm{~b} 2, \mathrm{~d} 8, \mathrm{~d} 11, \mathrm{~b} 3, \mathrm{c} 2, \mathrm{~d} 12$ \\
\hline 62. & मृ & $\mathrm{d} 2, \mathrm{~d} 3, \mathrm{~d} 4, \mathrm{~d} 5, \mathrm{~b} 2, \mathrm{~d} 8, \mathrm{~d} 11, \mathrm{~b} 3, \mathrm{c} 2$ & 87. & ग्रु & $\mathrm{d} 2, \mathrm{~d} 4, \mathrm{~d} 5, \mathrm{~b} 2, \mathrm{~d} 11, \mathrm{~b} 3, \mathrm{~d} 6, \mathrm{~d} 7$ \\
\hline 63. & ম্ব & $\mathrm{d} 2, \mathrm{~d} 3, \mathrm{~d} 4, \mathrm{~d} 5, \mathrm{~b} 2, \mathrm{~d} 12, \mathrm{~d} 8, \mathrm{~d} 11, \mathrm{~b} 3$ & 88. & স্ট & $\mathrm{a} 1, \mathrm{a} 2, \mathrm{~d} 2, \mathrm{~d} 4, \mathrm{~d} 5, \mathrm{~b} 2, \mathrm{~b} 3, \mathrm{~b} 10, \mathrm{~b} 9$ \\
\hline 64. & ম্ভ & $\mathrm{d} 2, \mathrm{~d} 3, \mathrm{~d} 4, \mathrm{~d} 5, \mathrm{~b} 2, \mathrm{n} 2, \mathrm{~d} 8, \mathrm{~d} 11, \mathrm{~b} 4, \mathrm{~b} 5$ & 89. & স্তু & $\mathrm{d} 2, \mathrm{~d} 4, \mathrm{~d} 5, \mathrm{~b} 2, \mathrm{n} 2, \mathrm{~d} 12, \mathrm{~b} 3, \mathrm{~b} 4, \mathrm{~b} 5$ \\
\hline 65. & म्म & $\mathrm{d} 2, \mathrm{~d} 3, \mathrm{~d} 4, \mathrm{~d} 5, \mathrm{~b} 2, \mathrm{~d} 7, \mathrm{~d} 10, \mathrm{~d} 9, \mathrm{~d} 11, \mathrm{~b} 3$ & 90. & ग्र & $\mathrm{d} 2, \mathrm{~d} 4, \mathrm{~d} 5, \mathrm{~b} 2, \mathrm{n} 2, \mathrm{~d} 12, \mathrm{b3}, \mathrm{b} 10, \mathrm{~b} 9$ \\
\hline 66. & म्ल & $\mathrm{d} 2, \mathrm{~d} 3, \mathrm{~d} 4, \mathrm{~d} 5, \mathrm{~b} 2, \mathrm{~d} 9, \mathrm{~d} 11, \mathrm{b3}$ & 91. & স্ন & $\mathrm{d} 2, \mathrm{~d} 4, \mathrm{~d} 5, \mathrm{~b} 2, \mathrm{~d} 11, \mathrm{~b} 3$ \\
\hline 67. & ল্ক & $\mathrm{a} 2, \mathrm{~d} 3, \mathrm{~d} 5, \mathrm{~b} 2, \mathrm{~d} 12, \mathrm{~d} 8, \mathrm{~d} 11, \mathrm{~b} 3, \mathrm{c} 2$ & 92. & न्भ & $\mathrm{d} 2, \mathrm{~d} 4, \mathrm{~d} 5, \mathrm{~b} 2, \mathrm{~d} 6, \mathrm{~d} 7, \mathrm{~d} 8, \mathrm{~d} 12, \mathrm{~b} 3$ \\
\hline 68. & ल্গ & a2,d3,d5,b2,d7,d10,d6,d12,b3 & 93. & न्य & $\mathrm{d} 2, \mathrm{~d} 4, \mathrm{~d} 5, \mathrm{~b} 2, \mathrm{~d} 7, \mathrm{~d} 11, \mathrm{~b} 3, \mathrm{c} 2$ \\
\hline 69. & ল্ট & a2,d3,d5,b2,b3,b10,b9,a1 & 94. & স্ব & $\mathrm{d} 2, \mathrm{~d} 4, \mathrm{~d} 5, \mathrm{~b} 2, \mathrm{~d} 12, \mathrm{~d} 8, \mathrm{~d} 11, \mathrm{~b} 3$, \\
\hline 70. & ल्ড & $\mathrm{a} 2, \mathrm{~d} 3, \mathrm{~d} 5, \mathrm{~b} 2, \mathrm{~d} 8, \mathrm{~d} 11, \mathrm{~b} 4, \mathrm{~b} 5$ & 95. & ग्म & $\mathrm{d} 2, \mathrm{~d} 4, \mathrm{~d} 5, \mathrm{~b} 2, \mathrm{~d} 12, \mathrm{~d} 8, \mathrm{~d} 11, \mathrm{~b} 3, \mathrm{c} 3, \mathrm{~b} 9, \mathrm{~b} 7$ \\
\hline 71. & ल्প & a2,d3,d5,b2,d8,d11,b3,d12,b8,b9 & 96. & স্ল & $\mathrm{d} 2, \mathrm{~d} 4, \mathrm{~d} 5, \mathrm{~b} 2, \mathrm{~b} 9, \mathrm{~d} 11, \mathrm{~b} 3$ \\
\hline 72. & ল্ম & $\begin{array}{l}\text { a2,d3,d5,b2,d8,d11,b3,d12,c3,b9, } \\
\text { b7 }\end{array}$ & 97. & रु & $\mathrm{a} 2, \mathrm{n} 1, \mathrm{~d} 1, \mathrm{~d} 4, \mathrm{~d} 7, \mathrm{~d} 11, \mathrm{~d} 5$ \\
\hline 73. & $\overline{् ্}$ & $\mathrm{a} 2, \mathrm{~d} 3, \mathrm{~d} 5, \mathrm{~b} 2, \mathrm{n} 2, \mathrm{~d} 8, \mathrm{~d} 11, \mathrm{~b} 4, \mathrm{~b} 5$ & & & \\
\hline
\end{tabular}

Here table 2 shown segment numbers for English alphabets (capital \& small) and table 3 shown segment numbers for Bangla \& English numerals.

Table 2: Segment Numbers For English Alphabets (capital \& small)

\begin{tabular}{|l|l|l|l|l|}
\hline $\begin{array}{c}\text { English } \\
\text { alphabet } \\
\text { (capital) }\end{array}$ & \multicolumn{1}{|c|}{ Segment numbers } & \multicolumn{1}{|c|}{$\begin{array}{c}\text { English } \\
\text { alphabets } \\
\text { (small) }\end{array}$} & \multicolumn{1}{|c|}{ Segment numbers } \\
\hline A & b1,b2,b3,b5,b6,d6,d12 & & a & b3,b4,,b5,b10,d6,d12 \\
\hline B & b1,b2,b3,b4,b5,b6,d6,d12 & & c & b3,b4,b5,,b6,d6,d12 \\
\hline C & b1,b4,b5,b6 & & d & b4,b5,d6,d12 \\
\hline D & b1,b2,b3,b4,b5,b6 & & e & b2,b3,b4,b5,d6,d12 \\
\hline E & b1,b4,b5,b6,d6,d12 & & g & b4,b5,d3,d5,d6,d12 \\
\hline F & b1,b5,b6,d6 & & h & b1,b2,b3,b4,b6,d10 \\
\hline G & b1,b3,b4,b5,b6,b8,b9 & & i & b5,b6,d9,d11 \\
\hline H & b2,b3,b5,b6,d6,d12 & & j & b3 \\
\hline I & a2,b2,b3,b4,d11 & & k & b1,b2,b3,d8,d11 \\
\hline J & b1,b2,b3,b4,b5,d6,d8 & & b5,b6,d8,d9,d11 \\
\hline K & b5,b6,d1,d4,d7,d11 &
\end{tabular}


Displaying All Bangla Compound Letters \& Alphabets By 32-Segment

\begin{tabular}{|c|c|c|c|}
\hline $\begin{array}{c}\text { English } \\
\text { alphabet } \\
\text { (capital) }\end{array}$ & Segment numbers & $\begin{array}{c}\text { English } \\
\text { alphabets } \\
\text { (small) }\end{array}$ & Segment numbers \\
\hline $\mathrm{L}$ & $\mathrm{b} 4, \mathrm{~b} 5, \mathrm{~b} 6$ & 1 & b2,b3,b4,b10 \\
\hline M & $\mathrm{b} 2, \mathrm{~b} 3, \mathrm{~b} 5, \mathrm{~b} 6, \mathrm{~d} 1, \mathrm{~d} 2$ & $\mathrm{~m}$ & $\mathrm{~b} 3, \mathrm{~b} 5, \mathrm{~d} 6, \mathrm{~d} 8, \mathrm{~d} 12$ \\
\hline $\mathrm{N}$ & $\mathrm{b} 5, \mathrm{~b} 6, \mathrm{~b} 7, \mathrm{~b} 9, \mathrm{c} 3, \mathrm{~d} 2, \mathrm{~d} 5$ & $\mathrm{n}$ & $\mathrm{b} 3, \mathrm{~b} 8, \mathrm{~b} 9, \mathrm{~d} 12$ \\
\hline $\mathrm{O}$ & b1,b2,b3,b4,b5,b6 & o & b3,b4,b5,d6,d12 \\
\hline $\mathrm{P}$ & $\mathrm{b} 1, \mathrm{~b} 2, \mathrm{~b} 5, \mathrm{~b} 6, \mathrm{~d} 6, \mathrm{~d} 12$ & $\mathrm{p}$ & b1,b2,b5,b6,d6,d12 \\
\hline $\mathrm{Q}$ & $\mathrm{b} 1, \mathrm{~b} 2, \mathrm{~b} 3, \mathrm{~b} 4, \mathrm{~b} 5, \mathrm{~b} 6, \mathrm{c} 3$ & $\mathrm{q}$ & $\mathrm{b} 3, \mathrm{~b} 10, \mathrm{~d} 3, \mathrm{~d} 5, \mathrm{~d} 6, \mathrm{~d} 12$ \\
\hline $\mathrm{R}$ & $\mathrm{b} 1, \mathrm{~b} 2, \mathrm{~b} 5, \mathrm{~b} 6, \mathrm{~d} 6, \mathrm{~d} 7, \mathrm{~d} 11, \mathrm{~d} 12$ & $\mathrm{r}$ & $\mathrm{b5}, \mathrm{d} 6, \mathrm{~d} 8$ \\
\hline $\mathrm{S}$ & $\mathrm{b} 1, \mathrm{~b} 3, \mathrm{~b} 4, \mathrm{~b} 6, \mathrm{~d} 6, \mathrm{~d} 12$ & $\mathrm{~s}$ & b4,d6,d7,d11,d12 \\
\hline $\mathrm{T}$ & $\mathrm{a} 2, \mathrm{~b} 2, \mathrm{~b} 3$ & $\mathrm{t}$ & b2,b3,b8,b10,d12 \\
\hline $\mathrm{U}$ & $\mathrm{b} 2, \mathrm{~b} 3, \mathrm{~b} 4, \mathrm{~b} 5, \mathrm{~b} 6$ & $\mathrm{u}$ & b3,b4,b5,b10 \\
\hline $\mathrm{V}$ & $\mathrm{b} 7, \mathrm{~b} 9, \mathrm{c} 3, \mathrm{~d} 2, \mathrm{~d} 5$ & $\mathrm{v}$ & $\mathrm{b} 5, \mathrm{~d} 8, \mathrm{~d} 10$ \\
\hline $\mathrm{W}$ & $\mathrm{b} 2, \mathrm{~b} 3, \mathrm{~b} 5, \mathrm{~b} 6, \mathrm{~d} 9, \mathrm{~d} 11$ & $\mathrm{w}$ & b3,b5,d9,d11 \\
\hline $\mathrm{X}$ & $\mathrm{d} 1, \mathrm{~d} 2, \mathrm{~d} 3, \mathrm{~d} 5$ & $\mathrm{x}$ & $\mathrm{d} 7, \mathrm{~d} 8, \mathrm{~d} 10, \mathrm{~d} 11$ \\
\hline $\mathrm{Y}$ & $\mathrm{b} 2, \mathrm{~b} 3, \mathrm{~b} 4, \mathrm{~b} 5, \mathrm{~d} 6, \mathrm{~d} 12$ & $\mathrm{y}$ & $\mathrm{b} 2, \mathrm{~b} 3, \mathrm{~d} 2, \mathrm{~d} 5, \mathrm{~d} 8, \mathrm{~d} 11$ \\
\hline $\mathrm{Z}$ & $\mathrm{b} 1, \mathrm{~b} 2, \mathrm{~b} 4, \mathrm{~b} 5, \mathrm{~d} 6, \mathrm{~d} 12$ & $\mathrm{z}$ & $\mathrm{b} 4, \mathrm{~d} 6, \mathrm{~d} 8, \mathrm{~d} 10$ \\
\hline
\end{tabular}

Table 3: Segment Numbers for Bangla \& English Numerals

\begin{tabular}{|l|l|l|l|l|}
\hline $\begin{array}{c}\text { English } \\
\text { numerals }\end{array}$ & \multicolumn{1}{|c|}{ Segment numbers } & \multicolumn{1}{|c|}{$\begin{array}{c}\text { Bangla } \\
\text { numerals }\end{array}$} & \multicolumn{1}{|c|}{ Segment numbers } \\
\hline 1 & $\mathrm{~b} 5, \mathrm{~b} 6$ & & $\mathrm{~S}$ & $\mathrm{~b} 1, \mathrm{~b} 2, \mathrm{~b} 3, \mathrm{~b} 4, \mathrm{~d} 9, \mathrm{~d} 11$ \\
\hline 2 & $\mathrm{~b} 1, \mathrm{~b} 2, \mathrm{~b} 4, \mathrm{~b} 5, \mathrm{~d} 6, \mathrm{~d} 12$ & & $\mathrm{~V}$ & $\mathrm{~b} 1, \mathrm{~b} 2, \mathrm{~b} 4, \mathrm{~b} 5, \mathrm{~d} 6, \mathrm{~d} 12$ \\
\hline 3 & $\mathrm{~b} 1, \mathrm{~b} 2, \mathrm{~b} 3, \mathrm{~b} 4, \mathrm{~d} 12$ & & $\checkmark$ & $\mathrm{b} 1, \mathrm{~b} 2, \mathrm{~b} 3, \mathrm{~b} 4, \mathrm{~b} 5$ \\
\hline 4 & $\mathrm{~b} 2, \mathrm{~b} 3, \mathrm{~b} 6, \mathrm{~d} 6, \mathrm{~d} 12$ & & 8 & $\mathrm{~b} 1, \mathrm{~b} 2, \mathrm{~b} 3, \mathrm{~b} 4, \mathrm{~b} 5, \mathrm{~b} 6, \mathrm{~d} 6, \mathrm{~d} 12$ \\
\hline 5 & $\mathrm{~b} 1, \mathrm{~b} 3, \mathrm{~b} 4, \mathrm{~b} 6, \mathrm{~d} 6, \mathrm{~d} 12$ & & $\varangle$ & $\mathrm{b} 1, \mathrm{~b} 2, \mathrm{~b} 3, \mathrm{~b} 4, \mathrm{~b} 5, \mathrm{~b} 6, \mathrm{~b} 8, \mathrm{~b} 9, \mathrm{~b} 10$ \\
\hline 6 & $\mathrm{~b} 1, \mathrm{~b} 3, \mathrm{~b} 4, \mathrm{~b} 5, \mathrm{~b} 6, \mathrm{~d} 6, \mathrm{~d} 12$ & & $\mathrm{~b} 2, \mathrm{~b} 3, \mathrm{~b} 4, \mathrm{~b} 5, \mathrm{~b} 6, \mathrm{~b} 8, \mathrm{~b} 9, \mathrm{~b} 10$ \\
\hline 7 & $\mathrm{~b} 1, \mathrm{~b} 2, \mathrm{~b} 3$ & $\mathrm{q}$ & $\mathrm{b} 1, \mathrm{~b} 2, \mathrm{~b} 3, \mathrm{~b} 6, \mathrm{~d} 6, \mathrm{~d} 12$ \\
\hline 8 & $\mathrm{~b} 1, \mathrm{~b} 2, \mathrm{~b} 3, \mathrm{~b} 4, \mathrm{~b} 5, \mathrm{~b} 6, \mathrm{~d} 6, \mathrm{~d} 12$ & $\mathrm{~b}$ & $\mathrm{~b} 5, \mathrm{~b} 6, \mathrm{~d} 6, \mathrm{~d} 8, \mathrm{~d} 10, \mathrm{~d} 12$ \\
\hline 9 & $\mathrm{~b} 1, \mathrm{~b} 2, \mathrm{~b} 3, \mathrm{~b} 6, \mathrm{~d} 6, \mathrm{~d} 12$ & & $\mathrm{~b} 1, \mathrm{~b} 2, \mathrm{~b} 3, \mathrm{~d} 8, \mathrm{~d} 12$ \\
\hline
\end{tabular}

\section{Result \& Discussion}

Different segmented display units are compared based on display quality, number of characters to be displayed, number of segments, and average active segments. Display quality is considered as main criterion because it plays vital role for acceptability of the display unit.

By comparing with other published paper we found some paper only for vowels or only consonant or only some compound letters, few paper for both vowel and consonant and English alphabets too but display quality not fair or have design complexity. In our paper we try to make a segment that has all things like vowel, consonant, numeral, compound letters and also good display. Here table 4 shown comparisons between our proposed design and previous published design. 
Table 4: Comparison Table between Our Proposed Design \& Published Design

\begin{tabular}{|l|l|l|}
\hline \multicolumn{1}{|c|}{ Number of segments } & \multicolumn{1}{|c|}{ Number of characters to be display } & Display Quality \\
\hline $9[1]$ & 10 ( only Bangla numerals) & Fair \\
\hline $10[2]$ & 10 ( only Bangla numerals) & Good \\
\hline $10[3]$ & 20 ( Bangla \& English numerals) & Good \\
\hline $11[4]$ & 10 ( only Bangla numerals) & Good \\
\hline $12[5]$ & 10 ( only Bangla numerals) & Good \\
\hline twin $7[6]$ & 10 ( only Bangla numerals) & Good \\
\hline $14[7]$ & 11 (only Bangla vowels) & Fair \\
\hline $17[8]$ & 11 (only Bangla vowels) & Fair \\
\hline $44[9]$ & 39 (only consonant) & Good \\
\hline $24[10]$ & 10 Bangla numerals, 11vowels,11 consonant & Fair \\
\hline $31[11]$ & 36 consonant & Poor \\
\hline $32[12]$ & 10 Bangla numeral, 50 bangla alphabets & Fair \\
\hline $26[13]$ & 50 Bangla alphabets & Fair \\
\hline $34[14]$ & 120 (Bangla, English, Arabic) & Fair \\
\hline $36[15]$ & 38 ( only bangla compound letters) & Good \\
\hline 32 (our proposed design) & 193 (all Bangla letters,numerals,compound letters and English & Good \\
\hline & letters,numerals) & \\
\hline
\end{tabular}

\section{Conclusion}

In this paper we have successfully used only 32 segments to represent all Bangla and English letters and numerals and also 97 compound letters. To our knowledge, it is the first segmented display design system for maximum Bangla compound letters. Our next effort will be try to reduce the number of segment, hardware implementation and more good looking display.

\section{References}

[1] Md. Badrul Alam Miah, Rashed Mazumder, S.M. Anamul Haque, and Nazrul Islam; "A NEW APPROACH TO DESIGN 9 SEGMENTS DISPLAY FOR BANGLA NUMERALS”, Canadian Journal on Electrical and Electronics Engineering Vol. 2, No. 12, December 2011

[2] Md. Abul Kalam Azad, Rezwana Sharmeen, Shabbir Ahmad and S. M. Kamruzzaman; "A Unique 10 Segment Display for Bengali Numerals",

[3] Sabbir Ahmed, Serazam Monira; "Designing a 10 segment display for Bangla and English numerals", 6th ICCIT 2004

[4] Ahmed Yousuf Saber, Mamun Al Murshed Chowdhury, Suman Ahmed, Chowdhury Mofizur Rahman; "Designing 11-Segment Display for Bangla Digit"s, 5th ICCIT 2002

[5] Al-Sakib Khan Pathan ,Md. Mahbub Alam, Mostafa Monowar, Forhad Rabbi, Sabbir Ahmed,Tareq Hasan Khan; "12-SEGMENT DISPLAY FOR BENGALI NUMERICAL CHARACTERS”, National Conference on Computer Processing of Bangla (NCCPB-2004), pp. 82-88, Dhaka, Bangladesh, February, 2004

[6] Gahangir Hossain and A. H. M. Ashfak Habib , 2004. "Designing Twin 7-Segment Display for Bangla Numeric Characters” . Asian Journal of Information Technology, 3: 49-55

[7] salahuddin mohammad masum,Mohammad Abdullah Al Mamun; "Designing 14-segment display for bengali vowels", Journal of Information technology 4(2): 178-184,2005

[8] ISLAM, A.K.M.N., MAHMUD, S.M.M., SHAHRIER, N., \& SATTAR, M.A.(2003); "Designing 17-segment Display for Bangla Vowels", 6th ICCIT 2003,Jahangirnagar University, PP. 283-286, December 19-21.

[9] MASUM, S.M., HAQUE, S.M., DASH, S.C., \& KABIR, K.F. (2005); "Segmented Display System for Bengali Consonants", Asian Journal of Information Technology, Grace Publications Network, Vol. 4 No. 2, PP. 291-300, 2005.

[10] Mohammad Shamsul Arefin, Mohammad Ali Akber Dewan, Muhammad Ibrahim Khan and Md. Shahidul Islam; "DESIGNING A 24SEGMENT DISPLAY FOR BENGALI NUMERICAL DIGITS AND CHARACTERS", 3rd International Conference on Electrical \& Computer Engineering ICECE 2004, 28-30 December 2004, Dhaka, Bangladesh

[11] MAHMUD, S., AREFIN, A.S., \& KHAN, M.I. (2005); "A New Approach for Displaying All Bengali Characters", Asian Journal of Information Technology, Grace Publications Network, Vol. 4 No. 2, PP.194-197

[12] Tamanna Haque Nipa, Md. Rakib Hasan, Nasrin Akhter; “32 Segment Display for Bengali Alphanumeric Characters”, International Journal of Science and Engineering Investigations vol. 2, issue 15, April 2013

[13] Mohammad Osiur Rahman, Mohammad Aktaruzzaman Khan, "Display Unit for Bangla Characters"; IIUC STUDIES ISSN 1813-7733 Vol. 4, December 2007 Published in April 2008 (p 71-86)

[14] Fariha Tasmin Jaigirdar, Mohammad Abdul Hadi Bhuiyan, Shahid Al Noor; "Sustainable Approach to Segmented Digital Display:Complete, Precise and Economic", Proceedings of 13th International Conference on Computer and Information Technology (ICCIT 2010)23-25 December, 2010, Dhaka, Bangladesh

[15] Md. Shahnur Azad Chowdhury, Ms Sultana Akter and Md Aman Ullah; “ Designing a 36 Segment Display for Bengali Compound Character", IJCSNS International Journal of Computer Science and Network Security, VOL.13 No.3, March 2013

[16] Tanzin Rahman, Tanvir Khan, Sarder Saadat Ahmed , Chandan Kumar Karmakar, "N-Segmented Display of Bangla Numerals", $8^{\text {th }}$ ICCIT,2005

[17] Bangla to English Dictionary, Bangla Academy 